\title{
Análisis socioeconómico en certificación globalgap de productores bananeros de la asociación fincas de el oro 2015-2019
}

\section{Socio-economic analysis in globalgap certification of banana producers of the fincas de el oro association 2015-2019}

\author{
DOI: $10.46932 / \mathrm{sfjdv2n4-047}$
}

Received in: March 1st, 2021

Accepted in: May 30th, 2021

Katherine González-Cruz

Estudiante de la Carrera Economía Agropecuaria, Universidad Técnica de Machala, Facultad de

Ciencias Agropecuarias, Ecuador.

E-mail:kbgonzalez est@utmachala.edu.ec

Jessica Quezada Campoverde

Universidad Técnica de Machala, Facultad de Ciencias Agropecuarias, Ecuador.

E-mail:jquezada@utmachala.edu.ec

Hector Ramiro Carbajal Romero

Universidad Técnica de Machala, Facultad de Ciencias Agropecuarias, Ecuador.

E-mail:hcarbajal@utmachala.edu.ec

Salomón Alejandro Barrezueta Unda

Universidad Técnica de Machala, Facultad de Ciencias Agropecuarias, Ecuador.

E.mail:sabarrezueta@utmachala.edu.ec

\section{Ángel Eduardo Luna Romero}

Universidad Técnica de Machala, Facultad de Ciencias Agropecuarias, Ecuador.

\section{RESUMEN}

En el presente artículo, el estudio de los perfiles socioeconómicos de los agricultores en Ecuador es escasos, por esta razón, la presente investigación busca como objetivo describir el análisis socioeconómico de la Asociación Fincas de El Oro en la implementación de la certificación Globalgap de los productores bananeros durante el periodo 2015-2019. La población estuvo conformada por los 45 asociados, los cuales respondieron un cuestionario estructurado por preguntas cerradas, que conforman los indicadores sociales, económicos. En la investigación se aplicó un diseño no experimental y de enfoque mixto. Los cuestionarios aplicados fueron sometidos a un control de calidad, para proceder a codificar y transcribir los datos en formato Excel, donde se utilizó una estadística descriptiva para verificar los resultados. Los resultados alcanzados establecen en general, en el rango de edad de los agricultores masculino se evidencian una gran participación existen de 45 hasta 56 años que se dedican a la producción de banano, mientras que el género femenino se observan intervalos de 69 hasta 82 años. Los asociados de Machala y Arenillas cuentan con mayores estudios en Secundaria y Universitaria. La mayoría de los asociados contestaron que no disponen de energía eléctrica, agua potable y telefonía fija. Se evidencia que más de la mitad de los trabajadores cuentan con seguro social. Una mayor productividad entre los asociados es Machala, en comparación con Arenillas, Santa Rosa y El Guabo. Siendo productores de la ciudad de Pasaje con menores ingresos a 2000 dólares americanos y productividad por hectárea de 1285 cajas/año. 
Palabras clave: Productividad agrícola, certificación, ingresos, servicios básicos.

\begin{abstract}
In this article, the study of the socioeconomic profiles of farmers in Ecuador is scarce, for this reason, this research aims to describe the socioeconomic analysis of the Fincas de El Oro Association in the implementation of the Globalgap certification of banana producers during the period 2015-2019. The population consisted of the 45 associates, who answered a questionnaire structured by closed questions, which made up the social, economic indicators. The research applied a non-experimental design and a mixed approach. The questionnaires applied were subjected to quality control, in order to proceed to code and transcribe the data in Excel format, where descriptive statistics were used to verify the results. The results obtained establish that in general, in the age range of the male farmers, there is a high participation from 45 to 56 years of age who are engaged in banana production, while in the female gender there are ranges from 69 to 82 years of age. The associates from Machala and Arenillas have more secondary and university education. The majority of the members answered that they do not have electricity, potable water or landline telephony. It is evident that more than half of the workers have social security. A higher productivity among the associates is Machala, compared to Arenillas, Santa Rosa and El Guabo. Producers in the city of Pasaje have a lower income of 2000 US dollars and productivity per hectare of 1285 boxes/year.
\end{abstract}

Key words: Agricultural productivity, certification, income, basic services.

\title{
1 INTRODUCCIÓN
}

La agricultura orgánica despuntó con fuerza en la década de los 80 , soportada en una serie de elementos integrales de manejo de los sistemas de cultivo e involucrando elementos técnicos, sociales, económicos y agroecológicos, que pretendían disminuir la dependencia a los insumos externos y los impactos ambientales, proporcionando alimentos saludables a mercados altamente competitivos y exigentes. Pero también debe abordar desafíos en materia de seguridad alimentaria, equidad e inclusión de género y la reducción de la pobreza rural (Mwendia, 2019).

Todos ellos tienen características similares; es decir, son globales, son complejos y están interrelacionados. En este contexto, muchas situaciones sociales y económicas por las que atraviesan los agricultores en distintas regiones del planeta comparten características similares como: desigualdad de género en el reparto de los recursos, bajo nivel de formación formal (escolaridad), ausencia de los servicios básicos, bajo salarios, escaso control del mercado local, etc. (Mwendia, 2019; Sato et al., 2018).

Las percepciones de estas similitudes surgen de estudios sobre el cambio en los modelos de producción dentro de los sistemas agrarios, la escasez de recursos naturales como el agua para el riego, entre otros factores. Por ende, está ligado de forma directa o indirecta con el ambiente (Oya et al., 2017).

Una característica socioeconómica en general, es acceder a créditos del sistema bancario; problema que afecta de forma directa a las dimensiones económicas, sociales y ambientales. En múltiples 
ocasiones los pequeños o medianos productores de banano no alcanzan los requerimientos del mercado internacional por la baja calidad de la fruta (Bucała-Hrabia, 2017; López \& Vargas, 2017).

Por ello se considera necesario adoptar estrategias asociativas e insertarse en una asociación para mejorar el nivel de producción y alcanzar los estándares de calidad comercial y ambiental requeridos. Para abordar las dimensiones sociales, económicas de los agricultores es necesario describir un perfil, que indique las características particulares y generales que los diferencian (Mwendia, 2019).

Los sistemas productivos agroalimentarios a nivel internacional han adoptado nuevas normas en la calidad del producto es por esta razón que en Europa surge la certificación Globalgap (Good Agricultural Practice), como una iniciativa enfocada en el sector minorista británico con el objetivo de tomar conciencia en la inocuidad alimentaria, el impacto ambiental, salud y seguridad de los trabajadores, calidad de agua en el riego, instalaciones sanitarias, control de plagas, gestión de residuos peligrosos. Las Buenas prácticas agrícolas dieron la creación a EUREPGAP en el año de 1987, la cual se enfocó en desarrollar normas y procedimientos tomando importancia a gran escala mundial (Zamora, 2020).

Ecuador es uno de los países más ricos en producción agroalimentaria y representa el $28 \%$ del producto interno bruto, es un motor en el desarrollo del crecimiento económico y social ya que satisface una gran parte de la demanda a nivel mundial y nacional, la mayor parte de su producción es exportada a países europeos y americanos teniendo una demanda muy elevada por lo cual tiene exigencias que cumplir y estándares internacionales, el país ha reconocido la necesidad de la sostenibilidad y la inocuidad alimentaria (Vite Cevallos et al., 2020).

Justamente, debido al auge alcanzado en el mercado internacional del consumo de productos orgánicos, en las últimas décadas se han potenciado los sistemas de certificación de estos productos para avalar las características que lo identifican. Estos sistemas de certificación ambiental garantizan la legitimidad de los productos orgánicos y de esta manera el consumidor final tiene la confianza de que el producto es inocuo y de calidad, otorgándole un valor agregado que aumenta su competitividad (Silva \& Brain, 2016).

Benítez (2017) demuestran que las producciones orgánicas que están certificadas obtienen mayores beneficios económicos, reciben facilidades para acceder a créditos, capacitación sobre este tipo de actividad, precios competitivos. Sin embargo, la utilización de la certificación como una herramienta para elevar la competitividad de los productos orgánicos se ve limitada, porque muchas veces los productores carecen de recursos económicos, falta de información no solo sobre las agencias de certificación sino, sobre cómo se desarrolla el proceso. 
De esta manera se desprende que el objetivo de la presente investigación es describir el análisis socioeconómico de la Asociación Fincas de El Oro en la implementación de la certificación Globalgap de los productores bananeros durante el periodo 2015-2019.

\section{CULTIVO DE BANANO Y SU PRODUCCIÓN EN ECUADOR}

El banano (Musa AAA cv. Grande Naine, Valery y Williams) es uno de los cinco cultivos alimenticios más importantes del mundo, después del trigo, el arroz, el maíz y la papa, y Ecuador es un importante productor de banano y el principal exportador de banano del mundo (López \& Vargas, 2017). El banano para el país, representa casi el 25\% de la producción de producto nacional bruto agrícola. En 2014 se exportaron 286 millones de cajas de $19.5 \mathrm{~kg}$, producidas en 266124 ha, lo que arrojó un ingreso total de US \$2300 millones FOB y genera fuente de trabajo para aproximadamente 2.5 millones de personas (Cabrera et al., 2020).

La producción y comercialización mundial de banano ha experimentado un aumento en los últimos años, en el 2019 aumentó un 5 por ciento en comparación del 2018 dando un lugar a Ecuador y Filipinas como los principales exportadores, dado que los mayores productores de banano lo realizan los pequeños productores agricultores es difícil obtener una cifra exacta en la demanda, a nivel mundial se consume más de 1000 variedades de banano y en su mayoría se contribuye a la seguridad alimentaria y un comercio justo (Soledispa et al., 2017).

La calidad al consumir un producto es un factor de mucha importancia para el consumidor ya que decidirá su compra. Las demandas en los supermercados internacionales antes de realizar su compra desean conocer si la fruta cumple todos los requisitos de calidad y seguridad alimentaria, que cada vez exigen los compradores (Jiménez et al., 2020).

La adopción de la implementación en la certificación Globalgap de los pequeños productores bananeros implica una inversión financiera mayor ya que esta norma exige que tengan una adecuada infraestructura en su unidad productiva y así cumpla con todas las medidas de bioseguridad y ambientales el plazo que dura la certificación es de doce meses (Caicedo-Camposano et al., 2020).

Las provincias más representativas son: Los Ríos, Guayas, y El Oro. Solo en la provincia de El Oro, hasta el año 2016 la superficie cosechada fue de 42340 ha, de las cuales, el 50\% correspondió a pequeños productores bananeros. De este segmento, el $80 \%$ de las fincas bananeras se manejan con una agricultura con altos insumos químicos, que los utilizan de forma excesiva (Villanueva et al., 2020).

\section{METODOLOGÍA}


El presente estudio se desarrolló en la provincia de El Oro, que está ubicada en el litoral sur del Ecuador, centrando la investigación en la Asociación Agraria Bananera Fincas de El Oro. Misma que está conformado por los 45 productores, con sus respectivos predios agrícolas, que tuvieron una superficie cultivada de entre 2,32 a 22.85 ha, en la Figura 1 se encuentran ubicados los productores de la Asociación Agraria Finca de El Oro. 
Figura 1. Ubicación de las fincas que se encuentran en diferentes cantones

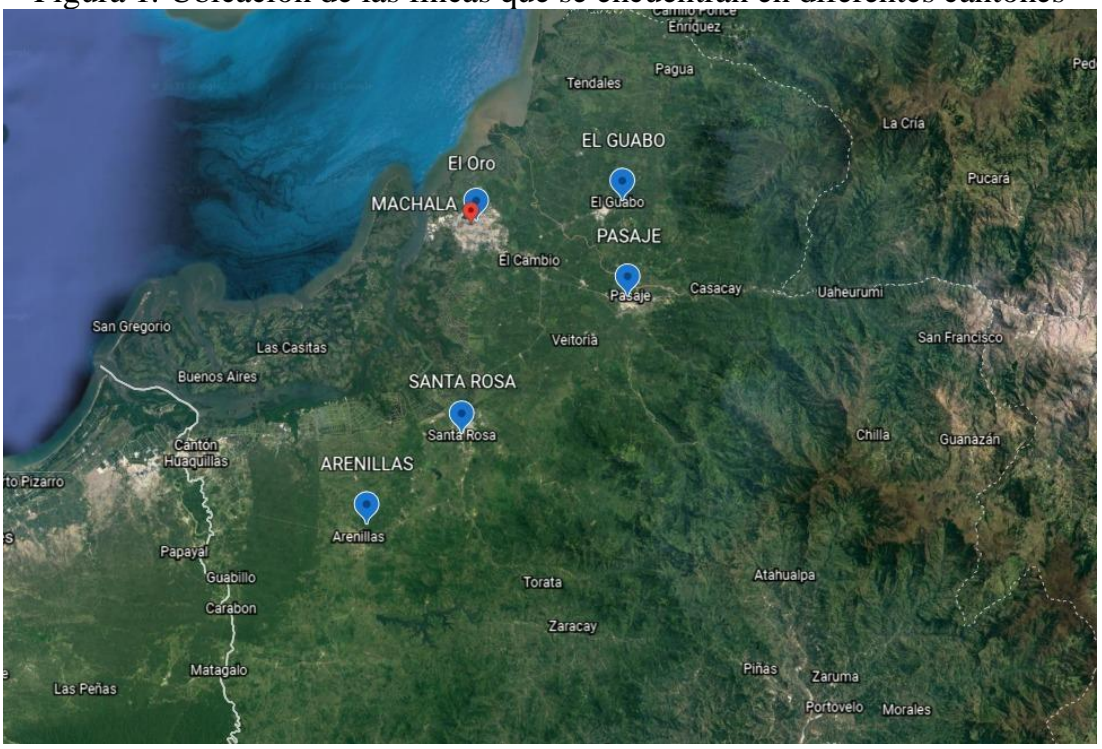

Fuente: google earth.

La presente investigación utilizó un diseño metodológico descriptivo y transversal, con el fin de analizar y describir el objetivo con variables categóricas y cuantitativas. Por lo cual, se elaboró un cuestionario conformado con preguntas abiertas y cerradas, que fue llenado de manera virtual haciendo uso de la herramienta de formularios de Google para evitar contacto físico con los encuestados.

La técnica empleada en el levantamiento de datos fue la encuesta, a través de un cuestionario que fue sometido a validación por 5 de expertos del sector bananero.

Para el desarrollo del estudio se diseñó un cuestionario de 2 ítems (Tabla 1) dirigido a los 45 productores de la Asociación Agraria Bananera Fincas de El Oro.

Tabla 1. Características de los indicadores por dimensión social y económica, empleado una encuesta a la Asociación Agraria Bananera Fincas de El Oro.

\begin{tabular}{|c|c|c|c|}
\hline \multirow[t]{2}{*}{$\mathbf{N}^{\circ}$} & \multicolumn{3}{|c|}{ Social } \\
\hline & Pregunta & Tipo de variable & Significado \\
\hline 1 & Género & Dicotómica & Femenino/Masculino \\
\hline 2 & Edad & Intervalo & $21-82$ años \\
\hline 3 & Nivel de instrucción & Nominal & Primaria; Secundaria; Universitaria; Ninguna \\
\hline 4 & Seguro Social & Dicotómica & $\mathrm{Si} / \mathrm{No}$ \\
\hline 5 & Servicio Básicos & Dicotómica & $\mathrm{Si} / \mathrm{No}$ \\
\hline \multirow[t]{2}{*}{$\mathbf{N}^{\circ}$} & \multicolumn{3}{|c|}{ Económico } \\
\hline & Pregunta & Tipo de variable & Significado \\
\hline 1 & Cantidad de Hectáreas & Intervalo & 1-6 ha; $6-10$ ha; $11-20$ ha;>20 ha \\
\hline 2 & Productividad & Intervalo & $\begin{array}{l}\text { Cosecha: } 100-300 \quad \text { racimos/ha } 301-500 \\
\text { racimos/ha;> 501 racimos/ha. }\end{array}$ \\
\hline 3 & Financiamiento & Nominal & $\begin{array}{l}\text { Banca Privada, Banca pública, Cooperativas de } \\
\text { Ahorro y créditos. }\end{array}$ \\
\hline
\end{tabular}


4 Ingresos Intervalo 400 a $1.000 ; .1001$ a $1400 ; 1401$ a $2000 ;>2000$ dólares americanos.

Fuente: Indicadores modificados para la investigación; tomados de los trabajos de; Prado et al., (2019), Prado et al., (2020), y Barrezueta-Unda \& Paz, (2017).

\subsection{MÉTODO DE ANÁLISIS DE DATOS}

Los cuestionarios aplicados fueron sometidos a un control de calidad, para proceder a codificar y transcribir los datos en formato Excel, donde se utilizó métodos estadísticos para verificar los resultados obtenidos a través del cuestionario empleando en el programa estadístico IBM SPSS Statistics v. 24. Con base a los resultados obtenidos se elaboraron gráficas y tablas para su respectiva interpretación.

En el análisis de la información se tuvo en cuenta el comportamiento de cada variable particularizando por cada finca a que pertenecen los productores y de manera general, lo que permite una visión más realista acerca del comportamiento del objeto de estudio en cuestión.

De esta manera, la presente investigación se realizó en dos momentos secuenciales en el tiempo. En un primer momento, se utilizó la prueba del alfa de Cronbach para determinar la confiabilidad del instrumento. En un segundo momento, se aplicó estadística descriptiva que permitió analizar el comportamiento de las variables mediante los cálculos de porcentajes de participación, distribución de frecuencias, tablas y gráficos.

\section{RESULTADOS Y DISCUSIÓN}

La confiabilidad del instrumento se realizó una prueba piloto, el instrumento conformado por 9 preguntas con una prueba piloto de 20 encuestados. Con un resultado del estadígrafo de Alpha de Cronbach de 0.84 que establece que el modelo de encuesta es confiable. En investigaciones similares al estudio relacionadas con datos cualitativos, Barrezueta \& Paz (2018) y Prado et al., (2020) obtuvo un Alpha de 0,836 que recomienda el uso a partir de 0,70 por lo que se aceptó el modelo del cuestionario. Es decir, el instrumento tiene una confiabilidad alta y por ello nos servirá para poder recolectar los datos posteriormente.

Tabla 2. Resumen de las estadísticas de procesamiento y fiabilidad de los casos

\begin{tabular}{cccccc}
\hline & & N & $\%$ & & \\
\hline & Valido & 20 & 100 & Alfa de Cronbach & $\mathbf{N}^{\circ}$ de Elementos \\
\hline Casos & Excluidos & 0 & 0 & 0,84 & 9 \\
\hline & Total & 20 & 100 & & \\
\hline
\end{tabular}


Luego de realizar la agrupación de las variables de estudio, se obtuvo las frecuencias y porcentajes respecto a las respuestas elegidas por los 45 agricultores de la Asociación Agraria Bananera Fincas de El Oro.

En la Figura 1, se evidencia que la mayoría de agricultores de la Asociación Agraria Bananera Fincas de El Oro se encuentran en el cantón Machala y Santa Rosa, ambos con 16 productores, en términos relativos representa el (67\%), mientras que el (33\%) se encuentran Pasaje, Arenillas y El Guabo. Estos datos se los obtuvo mediante una encuesta.

Figura 1.) Participación de género de agricultores por cantón.

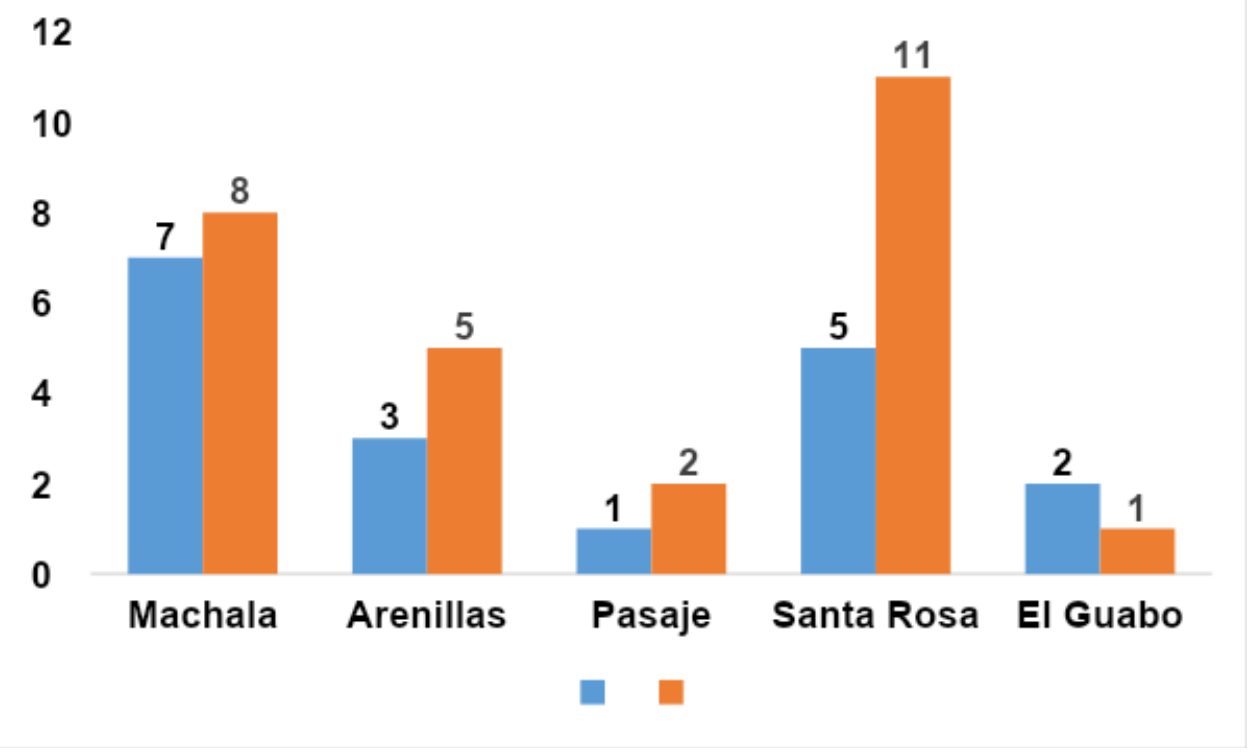

\subsection{INDICADORES SOCIALES}

En el análisis de los resultados, la relación género y edad se puede apreciar en la Tabla 3. En general, la población fue dominada por el género masculino (31) sobre el femenino (14); de forma relativa representa un $(31 \%)$ de la participación de la mujer entre los asociados. En el rango de edad de los agricultores masculino se evidencian una gran participación existen de 45 hasta 56 años que se dedican a la producción de banano, mientras que el género femenino se observan intervalos de 69 hasta 82 años. Sin embargo, según Prado (2019) la mujer está afianzándose en el área agropecuaria, es necesario que la mujer reciba capacitaciones en contabilidad, costos de producción y manejo ambientales en la producción bananera. 
Tabla 3. Participación de género y edad de los agricultores.

\begin{tabular}{|c|c|c|c|c|c|c|c|c|c|c|}
\hline \multirow[b]{2}{*}{ Edad } & \multicolumn{2}{|c|}{ Machala } & \multicolumn{2}{|c|}{ Arenillas } & \multicolumn{2}{|c|}{ Pasaje } & \multicolumn{2}{|c|}{ Santa Rosa } & \multicolumn{2}{|c|}{ El Guabo } \\
\hline & $\mathbf{M}^{1}$ & $\mathbf{H}^{2}$ & $\mathbf{M}^{\mathbf{1}}$ & $\mathbf{H}^{2}$ & $\underset{1}{\mathbf{M}}$ & $\mathbf{H}^{2}$ & $\mathbf{M}^{1}$ & $\mathbf{H}^{2}$ & $\mathbf{M}^{1}$ & $\mathbf{H}^{2}$ \\
\hline 21 a 32 & & & 1 & 1 & & & 1 & 2 & & \\
\hline 33 a 44 & & & 1 & 1 & & & 1 & 3 & & \\
\hline 45 a 56 & & 4 & & 2 & & 1 & 2 & 4 & 1 & \\
\hline 57 a 68 & 4 & 2 & & & & 1 & & 1 & & \\
\hline 69 a 82 & 3 & 3 & 1 & 2 & & & 1 & 1 & 1 & \\
\hline
\end{tabular}

${ }^{1}$ Mujer; ${ }^{2}$ Hombre

Se indica el nivel de instrucción formal por género, en la Figura 2, de acuerdo a la encuesta aplicada, la mayor frecuencia relativa fue el género masculino presenta más de la mitad $(68,90 \%)$ de personas que han culminado sus estudios. En cambio, el género femenino fue el de menor frecuencia relativa $(31,10 \%)$. Se visualiza que, del $100 \%$ de agricultores encuestados de Machala y Arenillas, $(71 \%)$ establecen contar con estudios finalizados en primaria, bachiller y universitaria. También al consultarle a otro grupo agricultores de los cantones Pasaje, Santa Rosa y El Guabo, ellos representan (29\%) de agricultores que han culminados sus estudios. Es importante señalar, que la mayoría personas que fueron encuestadas indicaron que han finalizado sus estudios, según Prado et al., (2018) establece que con el transcurso del tiempo la Asociación Agraria Bananera Fincas de El Oro se convertirá en una empresa muy destacada para el sector agropecuario, debido que la mayoría de los socios cuentan con estudios.

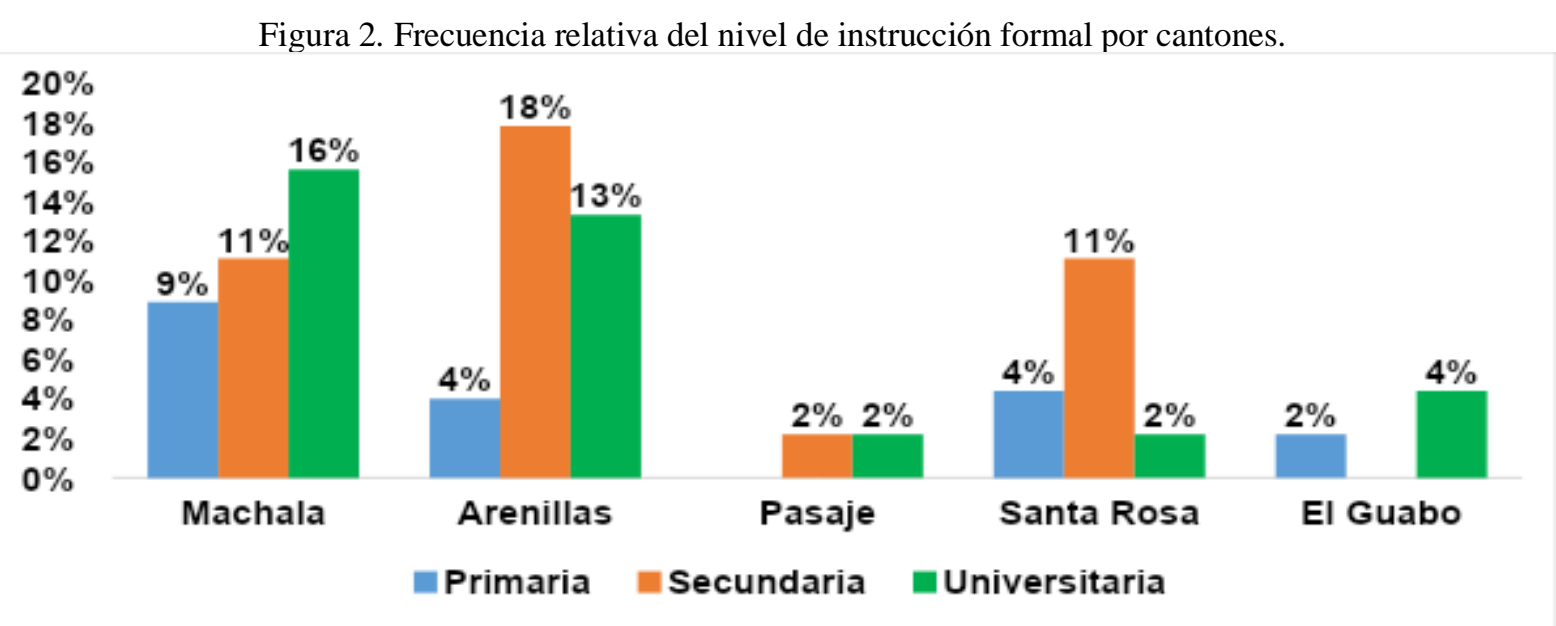

Se denota en la Tabla 4, que más de la mitad de los trabajadores (90\%) cuentan con seguro social, mientras que un (10\%) no disponen de un seguro social, esto como promedio en los diferentes cantones. Por esta razón, es necesario contar con un servicio de salud o algún Subcentro de salud en la comunidad. Según Ramírez et al., (2020) disponer de una atención primaria de salud y medicina integral permite garantizar de mejor manera la calidad de vida de la población. También podemos evidenciar que la 
mayoría de los asociados contestaron que no disponen de energía eléctrica, agua potable y telefonía fija. Sin embargo, se denota que el $(100 \%)$ de las personas cuentan con telefonía móvil.

Tabla 4. Frecuencia relativa de servicios básicos por cantones

\begin{tabular}{|c|c|c|c|c|c|c|c|c|c|c|c|c|c|c|}
\hline \multicolumn{15}{|c|}{ Afirmación (\%) } \\
\hline & \multicolumn{2}{|c|}{$\mathbf{S S}^{1}$} & \multicolumn{2}{|c|}{ EE } & \multicolumn{2}{|c|}{$\mathbf{A} \mathbf{P}^{3}$} & \multicolumn{2}{|c|}{$\mathbf{T F}^{4}$} & \multicolumn{2}{|c|}{$\mathbf{T} \mathbf{M}^{5}$} & \multicolumn{2}{|c|}{ IM $^{6}$} & \multicolumn{2}{|c|}{ IF7 } \\
\hline & $\mathrm{Si}$ & No & $\mathbf{S i}$ & No & $\mathbf{S i}$ & No & $\mathrm{Si}$ & No & $\mathbf{S i}$ & No & $\mathbf{S i}$ & No & $\mathbf{S i}$ & No \\
\hline Machala & 92 & 8 & 87 & 13 & 14 & 86 & 90 & 10 & 100 & 0 & 92 & 8 & 8 & 92 \\
\hline Arenillas & 89 & 11 & 58 & 42 & 12 & 88 & 32 & 67 & 100 & 0 & 91 & 9 & 6 & 94 \\
\hline Pasaje & 91 & 9 & 36 & 64 & 10 & 90 & 30 & 70 & 100 & 0 & 88 & 12 & 7 & 93 \\
\hline Santa Rosa & 90 & 10 & 34 & 66 & 12 & 88 & 25 & 75 & 100 & 0 & 86 & 14 & 5 & 95 \\
\hline El Guabo & 88 & 12 & 29 & 71 & 13 & 87 & 22 & 78 & 100 & 0 & 83 & 17 & 9 & 91 \\
\hline
\end{tabular}

${ }^{1}$ Seguro Social; ${ }^{2}$ Energía Eléctrica; ${ }^{3}$ Agua Potable; ${ }^{4}$ Telefonía Fija; ${ }^{5}$ Telefonía Móvil; ${ }^{6}$ Telefonía Móvil; ${ }^{7}$ Internet Fijo.

\subsection{INDICADORES ECONÓMICOS}

Como parte de los indicadores económicos tenemos cantidad de hectáreas (Tabla 5), productividad, ingresos y fuentes de financiamiento. Se observan intervalos de superficie cosechada por cantones, donde la mayoría de los asociados de Machala (42\%), Arenillas (35\%), Pasaje (51\%) y El Guabo (52\%), cuenta con 7 a 10 has $^{-1}$, seguido de las fincas entre 1 a 5 has $^{-1}$, donde el mayor valor fue registrado en Pasaje con un $78 \%$. Mientras que el intervalo $>20$ has $^{-1}$ tuvo con mayor proporción fue Arenillas con el $25 \%$ y El Guabo con un porcentaje de 25\%, coincidiendo con algunos trabajos realizados por Barrezueta et al., (2017) y Chungata et al., (2019) donde establecen que la mayoría de los asociados son pequeños agricultores (solo un 25\% es mayor a 20 ha en El Guabo). Esto se debe a que las certificaciones Globalgap y Orgánicas, certifican organizaciones que no difieran en grandes porciones entre las fincas más pequeñas con la más grande, igual sucede con la certificación Fairtrade.

Tabla 5. Relación relativa entre superficie por cantón

\begin{tabular}{lccccc} 
& Machala & Arenillas & Pasaje & Santa Rosa & El Guabo \\
\hline $\mathbf{1}$ a 6 has $^{-1}$ & $37 \%$ & $40 \%$ & $78 \%$ & $15 \%$ & $23 \%$ \\
$\mathbf{7}$ a $\mathbf{1 0}$ has $^{-1}$ & $42 \%$ & $35 \%$ & $22 \%$ & $51 \%$ & $52 \%$ \\
$\mathbf{1 1}$ a 20 has $^{-1}$ & $21 \%$ & & & $34 \%$ & $25 \%$ \\
\hline $\mathbf{2 0}$ has $^{-1}$ & & $25 \%$ & & & \\
\hline
\end{tabular}

Al analizar de forma general la productividad en las fincas (Tabla 6) se puede visualizar que Machala y Santa Rosa cuentan con mayor producción de racimo. Sin embargo, Pasaje y Machala no registran en sus fincas un >500 racimos, mientras que Santa Rosa es la que cuenta con mayor producción 
de >500 racimos. Esto se debe al buen manejo de labores culturales programadas que permiten mejorar una mayor producción de racimo por hectárea.

Tabla 6. Comparación absoluta entre los rangos de racimo procesados

\begin{tabular}{|c|c|c|c|c|c|}
\hline & Machala & Arenillas & Pasaje & Santa Rosa & El Guabo \\
\hline 100 a 300 racimo/ha & 8 & 3 & 4 & 5 & 2 \\
\hline 301 a 500 racimo/ha & 7 & 2 & 2 & 6 & 1 \\
\hline$>500$ racimo/ha & & 1 & & 3 & 1 \\
\hline Total & 15 & 6 & 6 & 14 & 4 \\
\hline
\end{tabular}

Se observa en la Tabla 7, la principal fuente de financiamiento provino de la banca pública, donde 23 asociados se beneficiaron de préstamos, también la banca privada realizó préstamos a 4 asociados, además 4 asociados recibieron dinero de otras fuentes. Finalmente, la cooperativa de ahorro y crédito otorgó crédito a 8 productores. Es necesario indicar que la mayoría productores recibieron préstamos por medio de la banca pública, siendo Machala y Santa Rosa los mayores.

Tabla 7. Comparación absoluta de fuentes de financiamiento

\begin{tabular}{lcccc}
\hline & Banca Pública & Banca Privada & $\begin{array}{c}\text { Cooperativa de ahorro } \\
\text { y crédito }\end{array}$ & Otras Fuentes \\
\hline Machala & 8 & 2 & 2 & 2 \\
Arenillas & 3 & 1 & & 1 \\
Pasaje & 2 & & 1 & \\
Santa Rosa & 9 & 1 & 5 & 1 \\
\hline El Guabo & 1 & & $\mathbf{8}$ & 4 \\
\hline Total & $\mathbf{2 3}$ & $\mathbf{4}$ & & \\
\hline
\end{tabular}

En la Tabla 8, se muestran los ingresos y producción por hectárea de banano por cantones que ha tenido la Asociación Agraria Bananera Fincas de El Oro en los últimos 5 años.

Se puede apreciar que productores del cantón Machala tienen una mejor productividad desde el 2015-2019 y mejores ingresos por concepto de ventas de cajas de banano por los mismos periodos.

Productores de Arenillas, Santa Rosa y El Guabo tienen similares producción e ingresos. Sin embargo, los productores de la ciudad de Pasaje tienen ingresos menores a 2000 dólares americanos y una productividad por hectárea de 1285 cajas/año.

Tabla 8. Análisis comparativo de rendimiento y ingresos por hectárea

\begin{tabular}{|c|c|c|c|c|c|c|c|c|c|c|}
\hline \multirow[b]{2}{*}{ Año } & \multicolumn{2}{|c|}{ Machala } & \multicolumn{2}{|c|}{ Arenillas } & \multicolumn{2}{|c|}{ Pasaje } & \multicolumn{2}{|c|}{ Santa Rosa } & \multicolumn{2}{|c|}{ El Guabo } \\
\hline & $\begin{array}{c}\text { Ingreso } \\
\mathrm{S}\end{array}$ & $\begin{array}{l}\text { Cajas } \\
22 X U\end{array}$ & $\begin{array}{c}\text { Ingreso } \\
\mathrm{S}\end{array}$ & $\begin{array}{l}\text { Cajas } \\
\text { 22XU }\end{array}$ & $\begin{array}{c}\text { Ingreso } \\
\mathrm{S}\end{array}$ & $\begin{array}{l}\text { Cajas } \\
22 X U\end{array}$ & $\begin{array}{c}\text { Ingreso } \\
\mathrm{S}\end{array}$ & $\begin{array}{l}\text { Cajas } \\
22 X U\end{array}$ & $\begin{array}{c}\text { Ingreso } \\
\mathrm{S}\end{array}$ & $\begin{array}{l}\text { Cajas } \\
22 X U\end{array}$ \\
\hline 2015 & 1731,57 & 1235 & 1632 & 1205 & 1645,12 & 1195 & 1631,57 & 1202 & 1631,57 & 1207 \\
\hline
\end{tabular}




\begin{tabular}{|c|c|c|c|c|c|c|c|c|c|c|}
\hline & & & & & & & & & \multicolumn{2}{|c|}{$\begin{array}{l}\text { SOUTTH FLORIDA } \\
\text { JOURAAL OF DEVELOPMENT }\end{array}$} \\
\hline 2016 & 1812,10 & 1250 & 1556,10 & 1232 & 1712,00 & 1201 & 1699,00 & 1228 & 1712,10 & 1230 \\
\hline 2017 & 2053,72 & 1389 & 1963,60 & 1302 & 1987,00 & 1287 & 1989,23 & 1279 & 1953,72 & 1285 \\
\hline 2018 & 2698,00 & 1392 & 2388,00 & 1341 & 2012,00 & 1305 & 2123,00 & 1336 & 2498,00 & 1340 \\
\hline 2019 & 2909,76 & 1450 & 2687,5 & 1389 & 2382,56 & 1365 & 2359,23 & 1352 & 2609,76 & 1365 \\
\hline Prom. & 2241,03 & 1343 & 2045,44 & 1294 & 1947,74 & 1271 & 1960,41 & 1279 & 2081,03 & 1285 \\
\hline
\end{tabular}

El nivel de ingresos de los asociados en la investigación, está en relación por producción hectárea, coincidiendo con planteado por Chungata et al., (2019) y Carpio et al., (2020) expresan que la conducta económica de los agricultores está inducida por la adquisición de maquinarias y equipo (estación de bombeo, motobombas, balanzas) y otros muebles e inmuebles (rodillos, escritorio, etc.,), como componentes de su principal actividad productiva.

\section{CONCLUSIÓN}

Se concluye en el análisis del perfil socioeconómico de la Asociación Agraria de Bananeros Fincas de El Oro, en general, la población fue dominada por el género masculino.

Los asociados de Machala y Arenillas cuentan con mayores estudios en Secundaria y Universitaria, además la mayoría de los asociados contestaron que no disponen de energía eléctrica, agua potable y telefonía fija.

Cabe señalar que más de la mitad de los trabajadores cuentan con un seguro social.

En este análisis se puede concluir que los asociados han mejorado su productividad y economía durante los últimos años, esto debido a la certificación Globalgap implementada ya que al adoptar las buenas prácticas agrícolas tenemos una acreditación en mercados internacionales que garantizan la calidad de la fruta. 


\section{BIBLIOGRAFÍA}

Barrezueta-Unda, S., \& González, A. P. G. P. (2017). Indicadores de sostenibilidad para la producción de cacao Nacional y CCN51 en la provincia El Oro-Ecuador. Educateconciencia, 13(14), 16-26. http://tecnocientifica.com.mx/educateconciencia/index.php/revistaeducate/article/download/152/21

Barrezueta, S. A. U., \& Paz-González, A. (2018). Indicadores de sostenibilidad sociales y económicos: Caso productores de cacao en El Oro, Ecuador. // Indicators of sustainability social and economic: Case cocoa farmers of El Oro, Ecuador. In CIENCIA UNEMI (Vol. 11, Issue 27, pp. 20-29). https://doi.org/10.29076/issn.2528-7737vol11iss27.2018pp20-29p

Benítez, C. L. B. C., \& Others. (2017). Diagnóstico de las certificaciones obtenidas por fincas productoras de banano orgánico en la provincia El Oro. Revista Científica Agroecosistemas, 5(1), 78-85. https://aes.ucf.edu.cu/index.php/aes/article/download/144/178

Bucała-Hrabia, A. (2017). Long-term impact of socio-economic changes on agricultural land use in the Polish Carpathians. Land Use Policy, 64, 391-404. https://doi.org/10.1016/j.landusepol.2017.03.013

Cabrera, J. B. Z., Guerrero, J. N. Q., \& Batista, R. M. G. (2020). La producción de banano en la Provincial de El Oro y su impacto en la agrobiodiversidad. Revista Metropolitana de Ciencias Aplicadas, 3(3), 189195. http://remca.umet.edu.ec/index.php/REMCA/article/view/327

Caicedo-Camposano, O., Soplín-Villacorta, H., Balmaseda-Espinosa, C., Cadena-Piedrahita, L., \& Leyva-Vázquez, M. (2020). Sustentabilidad de sistemas de producción de banano (Musa paradisiaca AAA) en Babahoyo, Ecuador. Revista Investigación Operacional, 41(3), 379-388. https://rev-invope.univ-paris1.fr/fileadmin/rev-inv-ope/files/41320/41320-07.pdf

Campoverde, A. E. C., Jiménez, K. E. C., Cevallos, H. A. V., \& Barrezueta-Unda, S. (2019). Perfil socioeconómico y ambiental de la asociación agraria bananera fincas de El Oro. Revista Metropolitana de Ciencias Aplicadas, 2(2), 182-190. http://remca.umet.edu.ec/index.php/REMCA/article/view/150

Jiménez, G. E. P., Zurita, I. N., \& Álvarez, J. C. E. (2020). Análisis del impacto tributario y contable por las variaciones del precio de la caja de banano en los productores del cantón Machala, Ecuador. Dominio de las Ciencias, 6(1), 396-428. https://dialnet.unirioja.es/servlet/articulo?codigo=7351796

López, E. V., \& Vargas, E. H. (2017). Análisis de la producción y la comercialización del banano como aspecto socio económico. Visionario Digital, $1(2), \quad 39-52$. https://doi.org/10.33262/visionariodigital.v1i2.241

Mwendia, A. S. (2019). An Investigation of the Drivers of Diversification to Banana Farming Among Households in Meru County, Kenya. Unpublished Masters of Arts In Geography. Kenyatta University, Nairobi. https://irlibrary.ku.ac.ke/bitstream/handle/123456789/19932/An\%20Investigation\%20of\%20the\%20Drivers....pd f? sequence $=1$

Oya, C., Schaefer, F., \& Skalidou, D. (2017). Effects of certification schemes for agricultural production on socio-economic outcomes in low-and middle-income countries: a systematic review. Campbell Systematic. https://onlinelibrary.wiley.com/doi/abs/10.4073/csr.2017.3 
Prado-Carpio, E., Castro-Armijos, C., Rentería-Minuche, P., Coronel-Reyes, J., Paladines-Amaiquema, J., \& Martínez-Soto, M. (2019). Agronegocio Conchero (Anadara tuberculosa): Aspectos socioeconómicos, Archipiélago de Jambelí. Cumbres, 5(2), 79-88. https://doi.org/10.48190/cumbres.v5n2a6

Ramirez, K. T., Castro, K. V., \& Reyes, J. C. (2020). Pronóstico de profesionales de la salud en la provincia del Oro, Ecuador. Polo del Conocimiento: Revista científico - profesional, 5(1), 542-562. https://dialnet.unirioja.es/servlet/articulo?codigo=7436043

Sato, Y., Komatsu, K., Kitanishi, K., Shikata-Yasuoka, K., \& Odani, S. (2018). Banana Farming, Cultivars, Uses, and Marketing of Nkore in Southwestern Uganda. Tropical Agriculture and Development, 62(3), 141-149. https://doi.org/10.11248/jsta.62.141

Serrano, L. A. L., Sisalima, M. F. A., Velásquez, N. A. B., \& Pineda, Y. L. B. (2020). Ecuador: Análisis comparativo de las exportaciones de banano orgánico y convencional e incidencia en la Balanza Comercial, 2018. Revista Científica y Tecnológica UPSE, 7(2), 38-46. https://doi.org/10.26423/rctu.v7i2.521

Soledispa, V. B. S., Salazar, G. M. D., \& Povea, R. A. A. (2017). El banano y su consumo en el Ecuador. Revista Publicando, $4(13 \quad$ 283-292. https://www.academia.edu/download/58318516/Consumo_de_banano_en_el_Ecuador.pdf

Villanueva, V. A., Correa, C. D. A., \& Bonisoli, L. (2020). Introducción de marca de banano orgánico en el mercado ecuatoriano. INNOVA Research Journal, 5(1), 166-183. https://doi.org/10.33890/innova.v5.n1.2020.1150

Vite Cevallos, H., Townsend Valencia, J., \& Carvajal Romero, H. (2020). Big Data e Internet de las Cosas en la producción de banano orgánico. Revista Universidad Y Sociedad, 12(4), 192-200. http://scielo.sld.cu/scielo.php?script=sci_arttext\&pid=S2218-36202020000400192

Zamora, G. V. (2020). Relaciones de género y condiciones laborales en el sector productivo del banano orgánico en Sullana, Piura. Investigaciones Sociales, 22(42), 315-327. https://doi.org/10.15381/is.v22i42.17496 